\section{Avaliação de uma metodologia de alocação de recursos financeiros do setor saúde para aplicação no Brasil}

\author{
Evaluation of a methodology for financial \\ resources allocation in Brazilian health sector
}

\author{
${ }^{1}$ Escola Nacional de Saúde \\ Pública Sergio Arouca, \\ Fundação Oswaldo Cruz, \\ Rio de Janeiro, Brasil. \\ 2 Centro de Informação \\ Científica e Tecnológica, \\ Fundação Oswaldo Cruz, \\ Rio de Janeiro, Brasil. \\ Correspondência \\ S. Porto \\ Departamento de \\ Administração e \\ Planejamento em Saúde, \\ Escola Nacional de Saúde \\ Pública Sergio Arouca, \\ Fundação Oswaldo Cruz. \\ Rua Leopoldo Bulhões 1480 , \\ Rio de Janeiro, $R J$ \\ 21041-210, Brasil. \\ sporto@ensp.fiocruz.br
}

\begin{abstract}
In the early 1990s, a group of British researchers developed a new methodology for healthcare resource allocation based on need. The methodology's main characteristics are to draw on the theoretical model for healthcare services demand and apply data on health services utilization to estimate needs-based use. The objective of the current study was to assess the applicability of this methodology for allocating Federal resources at the local level in Brazil. Data from all acute hospital admissions in 1999 came from the Inpatient Information System of the Unified National Health System (SUS). The country was divided into 134 geographic areas. The statistical models tested to estimate needs-based use applied the following need variables: infant mortality rate; standardized mortality rate; illiteracy rate; proportion of households headed by women; and mean number of household members. All tested models showed negative regression coefficients, indicating that the methodology is inadequate for resource allocation based on need in places like Brazil with large social inequalities in healthcare utilization.
\end{abstract}

Resource Allocation; Health Services Needs and Demand; Equity; Methodology
Silvia Porto ${ }^{1}$

Mônica Martins 1

Claudia Travassos 2

Francisco Viacava 2

\section{Introdução}

Diversos países têm realizado esforços para definir metodologias voltadas para orientar, de forma eqüitativa, a distribuição geográfica de recursos destinados ao financiamento do setor saúde. O conceito de eqüidade subjacente corresponde à igualdade na distribuição de recursos financeiros para necessidades de saúde iguais ${ }^{1}$. Em geral, essas experiências internacionais tratam separadamente a distribuição de recursos para custeio dos serviços de saúde e a distribuição de recursos destinados ao investimento. No que se refere à alocação de recursos para custeio, as abordagens metodológicas adotadas têm como referência as bases populacionais de cada área geográfica. A fórmula mais simples para alocação de recursos com base na população é a distribuição de recursos per capita. No entanto, essa abordagem desconsidera as diferenças existentes nas estruturas populacionais do ponto de vista demográfico e social, que impactam diretamente o perfil de necessidades de saúde. Assim, é importante a incorporação às fórmulas de alocação geográfica de recursos para custeio de medidas de necessidade de saúde, de forma a garantir maior eqüidade na sua distribuição. Essas medidas devem permitir dimensionar as desigualdades relativas entre as condições de saúde das populações de distintas áreas geográficas. É justamente na forma como são estimadas as necessidades de saúde que as abordagens diferenciam-se. As abordagens exis- 
tentes variam exatamente no grau de elaboração da metodologia empregada para dimensionar as necessidades de saúde relativas. Porém, dada a incomensurabilidade do construto necessidade de saúde, nenhuma das abordagens consegue dimensioná-lo em toda a sua extensão e complexidade.

Uma metodologia para alocação eqüitativa de recursos para custeio deve basear-se em sucessivos ajustes da base populacional, levando em conta o perfil demográfico, as diferenças entre os custos dos tratamentos requeridos por cada segmento de pacientes e as desigualdades entre as necessidades de saúde na população.

Dentre as fórmulas para alocação de recursos que incorporam as necessidades de saúde da população, destacam-se aquelas que adotam como medida de necessidade um único indicador, como, por exemplo, o coeficiente de mortalidade geral, padronizado por sexo e idade. Este é o caso da fórmula desenvolvida pelo Resource Allocation Working Party (RAWP), utilizada na Inglaterra entre 1976 e 1994, para a distribuição de uma parcela importante dos recursos financeiros destinados à prestação de cuidados de saúde 2 . O objetivo da fórmula RAWP é gerar uma distribuição inter-regional dos recursos financeiros que favoreça o alcance de maior igualdade de oportunidades no acesso aos serviços de saúde por necessidades de saúde iguais ${ }^{2}$. Essa fórmula tem a vantagem da simplicidade na sua aplicabilidade, além de ser de fácil compreensão, o que favorece sua aceitação pelos gestores. No entanto, implica assumir que um único indicador de saúde pode expressar a maior parte das necessidades da população. Esse pressuposto fez com que a formula RAWP se tornasse objeto de críticas que implicaram várias reformulações ao longo dos anos 3,4,5. A principal crítica refere-se ao questionamento da utilização da mortalidade padronizada como proxy de morbidade e, conseqüentemente, das necessidades de saúde da população ${ }^{6}$. As críticas apontam que as taxas de mortalidade padronizadas não expressam na sua totalidade as variações da morbidade da população de distintas áreas 7. O emprego da mortalidade como proxy de morbidade na fórmula RAWP é, no mínimo, considerado insuficiente ${ }^{8}$.

Partindo das críticas à formula RAWP, em 1994, pesquisadores da Universidade de York, Inglaterra 5 , elaboraram uma nova e mais refinada metodologia para a alocação de recursos de custeio com base nas necessidades de saúde. O que caracteriza essa metodologia é empregar dados sobre o uso de serviços de saúde para estimá-lo com base nas necessidades de saúde da população. A metodologia tem como referencial teóri- co o modelo de demanda aos serviços de saúde, cujos determinantes fundamentais são as necessidades de saúde da população e as características da oferta de serviços (Figura 1). Esse modelo assume também que características das pessoas não associadas às necessidades de saúde, tais como as expectativas dos indivíduos, influenciam a demanda aos serviços de saúde. Por outro lado, a oferta, ao mesmo tempo em que determina o uso de serviços, é por este influenciada, isto é, a oferta pode ser influenciada pelo uso de serviços em tempos anteriores, o que reflete o perfil de necessidade de saúde no passado, criando ao longo do tempo um feedback entre a oferta e a utilização de serviços de saúde. Nessa metodologia, o modelo de uso de serviços tem como variáveis independentes medidas diretas e indiretas (variáveis sociais) de condições de saúde, ajustadas pela oferta de serviços de saúde.

O objetivo deste artigo é avaliar a aplicabilidade no Brasil da metodologia proposta por Carr-Hill et al. 5 para a distribuição dos recursos financeiros do Ministério da Saúde entre as Unidades da Federação. No caso do Brasil, embora existam dados sobre uso de serviços de saúde, as desigualdades sociais existentes, tanto no que se refere à disponibilidade de serviços, como a outros fatores capacitantes do uso de serviços 9,10,11, são de magnitude muito superior às observadas na Inglaterra e em outros países, onde essa metodologia tem sido implementada ${ }^{12}$, o que pode vir a inviabilizar sua utilização.

\section{Metodologia}

A metodologia proposta por Carr-Hill et al. 5 foi testada para as internações de curta permanência (pacientes agudos) financiadas pelo Sistema Único de Saúde (SUS) no Brasil em 1999, utilizando-se os dados do Sistema de Informações Hospitalares do SUS (SIH-SUS). Os procedimentos médicos da Tabela de Procedimentos do SUS 13, que correspondem à unidade de pagamento do sistema de reembolso aos hospitais adotado pelo SUS, foram usados na seleção das internações de curta permanência. Nessa seleção foram ainda considerados a especialidade do procedimento, o diagnóstico principal e o tempo médio de permanência. Foram excluídas as internações psiquiátricas; aquelas codificadas como: "fora de possibilidade terapêutica", "cuidados prolongados", "internação domiciliar" e "reabilitação”. Além disso, excluíram-se também as internações obstétricas, considerando-se que, neste caso, o perfil de necessidade pode ser mais bem estimado a partir das taxas de fecundidade, estratégia também adotada na Inglaterra. 


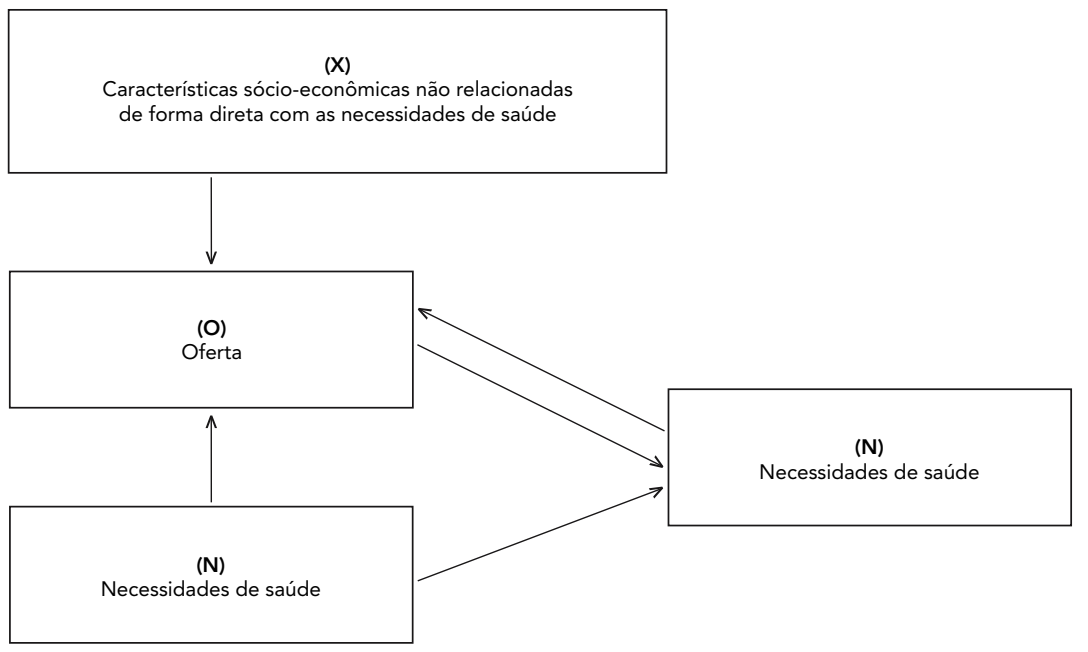

Fonte: Carr-Hill et al. 5

Analogamente ao caso inglês, considerouse necessário, para efeitos da modelagem estatística, definir unidades geográficas de análise menores do que as Unidades da Federação. A utilização do município como unidade geográfica de análise não foi viável devido ao fato de os municípios muito pequenos geralmente terem maiores deficiências na qualidade do registro das estatísticas vitais, apresentando instabilidade em vários indicadores. Um exemplo disso é a taxa de mortalidade ser bastante instável nestas localidades, já que poucos óbitos têm chance de ocorrer em um curto período de tempo. Além disso, os indicadores provenientes de dados das pesquisas amostrais de base populacional realizadas pelo Instituto Brasileiro de Geografia e Estatística (IBGE) são raramente disponíveis no âmbito do município. Dessa forma, foram selecionados como unidades geográficas de análise todos os municípios capitais dos 26 Estados, o Distrito Federal, os demais municípios com mais de 200 mil habitantes, e as áreas geográficas correspondentes ao conjunto dos demais municípios para cada Unidade da Federação. Foi assim constituído um banco de dados com 134 unidades geográficas.

A variável dependente no modelo de internações de curta permanência foi a razão entre o valor pago às internações realizadas pelos residentes de cada área e o valor estimado para as internações naquela área a partir dos valores médios nacionais. Utilizou-se o valor pago pela internação e não apenas o volume de internações, pois esse dado é mais completo na medida em que incorpora informação sobre a complexidade do atendimento. Isso porque, no caso do mecanismo de pagamento adotado pelo SUS, o valor pago às internações é função dos dias de permanência e do procedimento, e, em certos casos, de um conjunto de procedimentos realizados no paciente. $\mathrm{O}$ valor pago foi obtido agregando-se em cada área geográfica os valores pagos pelas internações realizadas pela população residente. O numerador da variável dependente foi obtido agregando-se para cada nível geográfico os valores pagos pelas internações de seus residentes. O denominador foi obtido aplicando-se as taxas médias nacionais e as médias nacionais dos valores pagos, segundo grupo de idade e sexo, à população residente em cada área geográfica. As faixas etárias adotadas foram: menores de 1 ano, 1 a 4 anos, 5 a 14 anos, 15 a 24 anos, 25 a 44 anos, 45 a 54 anos, 55 a 64 anos, e 65 anos e mais. Dessa maneira, a variável dependente pode ser entendida como uma razão do valor do uso observado pelo uso esperado, padronizada por idade e sexo, sendo, portanto, desnecessário incluir as variáveis idade e sexo na equação de uso de serviços de saúde, reduzindo-se, assim, o número de regressores no modelo de uso. 
As variáveis de oferta foram selecionadas a partir daquelas disponíveis no SIH-SUS e no censo de serviços de saúde, realizado pelo IBGE em 1999, denominado Assistência Médico-Sanitária (AMS) 14. Os indicadores empregados foram: o número de leitos privados sem vínculo com o SUS por 1.000 habitantes (AMS/1999); o número de leitos - públicos ou privados - com vínculo com o SUS por mil habitantes nas especialidades de clínica médica, cirurgia e pediatria (SIH-SUS / 1999); e o número de postos de trabalho médico em ambulatório por mil habitantes (AMS/1999). Postos de trabalho médico correspondem ao número de empregos médicos em serviços ambulatoriais, cujo somatório é superior ao total de médicos. A incorporação desta variável parte do pressuposto de que o número de empregos médicos influencia o volume de internações hospitalares financiadas pelo SUS em uma determinada área. Assumiu-se também que o volume de leitos privados sem vinculação com o SUS afeta a demanda aos leitos financiados pelo SUS. Calcularam-se também os indicadores de oferta ponderados pelo quadrado da densidade populacional. A correção das variáveis de oferta pela densidade populacional foi utilizada como forma de minimizar o efeito de possíveis discrepâncias entre a oferta e o tamanho da população na estimativa das necessidades associadas ao uso de serviços.

Características epidemiológicas, demográficas e sócio-econômicas da população foram incorporadas aos modelos como regressores no modelo de oferta e como variáveis proxy de necessidades no modelo de utilização de internações de curta permanência. As variáveis epidemiológicas empregadas nos modelos estatísticos foram: proporção de óbitos neonatais precoces; proporção de óbitos infantis mal definidos; proporção de óbitos infantis por diarréia e desnutrição; mortalidade proporcional por doença cardiovascular; mortalidade proporcional por doença infecto-parasitária; mortalidade proporcional por neoplasia; mortalidade proporcional por causa externa; coeficiente de mortalidade infantil; coeficiente de mortalidade geral padronizado por idade e sexo; taxa de óbitos na população de 65 anos e mais; taxa de incidência de AIDS de 1549 anos; e taxa de homicídio em homens de 15-49 anos. As demográficas foram: proporção de adolescentes; razão de dependência; percentual da população residente com 0-4 anos; percentual da população residente com 60-64 anos; percentual da população residente com 65 anos ou mais; e taxa de fecundidade. As sócio-econômicas foram: média de anos de estudos; percentual de analfabetismo; percentual de pessoas residentes em áreas urbanas; valor médio per capita de de- pósito bancário; percentual de pessoas ocupadas no mercado formal na própria área de residência; média de pessoas residentes em domicílios; percentual de domicílios particulares permanentes chefiados por mulheres. A variável "valor médio per capita de depósito bancário" não continha informação para um município - Ribeirão das Neves. Neste caso, utilizou-se o procedimento de imputação, usando-se o valor de referência do Município de Embú, localizado na mesma região geográfica e com número semelhante de habitantes para o ano de 1998.

Diante das limitações nas fontes primárias de estatística vital em grande parte dos municípios das regiões Norte, Nordeste e Centro-Oeste, foram utilizados procedimentos para estimar a mortalidade infantil e a mortalidade geral padronizada. Para corrigir essas limitações, o IBGE tem recorrido a técnicas demográficas, ditas de mensuração indireta, para substituir as estimativas clássicas. Tais técnicas são baseadas em dados de entrevistas domiciliares realizadas por ocasião dos censos demográficos ou das pesquisas nacionais por amostra de domicílio (PNAD).

Para a mortalidade infantil, os métodos de mensuração indireta baseiam-se em relacionar proporções de sobrevivência a medidas tradicionais de mortalidade, mediante a inclusão de perguntas de caráter retrospectivo nos censos e pesquisas domiciliares. Entretanto, em Estados que, reconhecidamente, têm sistemas de mortalidade com cobertura completa das informações de registro os resultados são obtidos por cálculo direto.

No presente trabalho, para estimar a mortalidade infantil para as 134 unidades geográficas, adotaram-se dois procedimentos. Quando a cobertura das informações de registro era considerada adequada, a mortalidade infantil foi estimada pelo método direto, utilizando-se os dados do Sistema de Informações sobre Mortalidade (SIM) e do Sistema de Informações de Nascidos Vivos (SINASC). Para os Estados e municípios com deficiência no registro dos dados vitais, foram utilizados fatores de correção obtidos por mensuração indireta do IBGE.

Para o cálculo da taxa de mortalidade padronizada por idade, optou-se por considerar como população-padrão a do Estado do Rio de Janeiro, por ser o Estado com a população mais idosa do Brasil. A taxa de mortalidade padronizada por idade é utilizada também para avaliar a cobertura das informações de registro de óbitos 15 . Valores inferiores a 6,8 por mil habitantes indicam falhas de registro. Nas unidades geográficas onde foram identificadas coberturas incompletas, as taxas de mortalidade foram corrigidas pelos fatores estimados pelo IBGE por mensuração indireta. 


\section{Análise dos dados}

Como apontado anteriormente, de acordo com o modelo teórico de demanda aos serviços de saúde, o uso de serviços é função das necessidades de saúde e da oferta de serviços. Esta, por sua vez, pode ter como determinantes as necessidades de saúde e o próprio uso. Nesse caso, tem-se o seguinte sistema de equações simultâneas, em que os valores de uso e oferta são determinados conjuntamente:

$$
\begin{aligned}
& U=f(N, O) \\
& O_{t}=f(N, U), t=1, \ldots, p
\end{aligned}
$$

onde: $N$ é um conjunto de variáveis demográficas, epidemiológicas e sociais ("instrumento"); $U$ é a variável que mede uso de serviços de saúde; $O$ é a variável de oferta de serviços de saúde; $N$ 'é um subconjunto de $\mathrm{N}$ (variáveis de necessidade); e $p$ é o número total de variáveis de oferta consideradas no modelo.

$\mathrm{O}$ "instrumento" $\mathrm{N}$, neste estudo, considerou todas as possíveis variáveis sociais e de saúde associadas à oferta de serviços, inclusive aquelas (N') associadas ao uso de serviços de saúde e que foram empregadas na equação de uso. Dessa forma, N' corresponde ao subconjunto das variáveis que compõem $\mathrm{N}$, que foram testadas como preditoras do uso de serviços.

A hipótese de existência de relação recíproca (causalidade reversa) entre o uso e a oferta de serviços de saúde aponta para a presença de endogeneidade. Caso confirmada a presença dessa relação, o estimador obtido através do Método de Mínimos Quadrados Ordinários (MQO) para os parâmetros das equações é tendencioso e inconsistente. Na presença de endogeneidade, o método estatístico adequado é a regressão em duas etapas. No presente estudo, este método foi empregado e as etapas realizadas foram: (i) regressão das variáveis de oferta por todas as variáveis sócio-econômicas, demográficas e epidemiológicas (modelo da oferta); (ii) regressão da variável do valor das internações ajustado pela oferta (modelo de uso) e (iii) regressão da variável do valor das internações sem ajuste da oferta. Os regressores desse modelo incluem apenas aqueles que se mostraram estatisticamente significativos no modelo de uso (modelo de predição da demanda).

Para testar a existência de endogeneidade, foram realizadas regressões para cada uma das variáveis de oferta. A equação genérica que expressa essas regressões é:

$$
O_{t}=f(N)+e_{t}
$$

onde: $N$ é um conjunto de variáveis demográficas, epidemiológicas e sociais ("instrumento"); e $e_{t}$ é o vetor de resíduos da equação t.
$\mathrm{O}$ vetor de resíduos $\left(e_{t}\right)$ estimado em cada uma das equações de oferta foi inserido como regressor na equação do modelo de uso [U = $\left.\mathrm{f}\left(\mathrm{N}^{\prime}, \mathrm{O}\right)\right]$.

Para testar a hipótese de endogeneidade da oferta, aplicou-se o teste $\mathrm{F}$ entre o modelo restrito (sem resíduos) de uso e o modelo irrestrito (com resíduos) de uso. A hipótese nula $\left(\mathrm{H}_{0}\right)$ refere-se à ausência de endogeneidade. A hipótese alternativa $\left(\mathrm{H}_{1}\right)$ considera que as variáveis de oferta são endógenas. O nível de significância adotado foi $95 \%$ (F tabular $=2,68$ ). Na presença de endogeneidade na equação de uso, foram utilizados os valores preditos obtidos nas equações de oferta em substituição dos valores observados das variáveis de oferta.

As variáveis que compõem o subconjunto N' (estimadores de necessidade associados ao uso de serviços) foram selecionadas a partir de $\mathrm{N}$ por critérios teóricos e empíricos, não excludentes entre si, com o objetivo de obter-se um modelo parcimonioso, capaz de discriminar desigualdades em saúde e de clara compreensão. Esses critérios foram: variáveis que contêm grande densidade de informação, como, por exemplo, mortalidade geral padronizada por idade e sexo priorizada em relação à mortalidade proporcional por grupos de causas; variáveis sociais sobre as quais existe evidência científica de associação positiva com as necessidades de saúde, por exemplo, "média de pessoas residentes em domicílios" 1; variáveis que se mostraram correlacionadas ao uso de serviços. Evitou-se a inclusão simultânea de duas ou mais variáveis correlacionadas entre si e excluíram-se variáveis epidemiológicas cuja ocorrência apresenta distribuição espacial concentrada em algumas áreas, como a taxa de incidência de AIDS. Foram selecionadas as seguintes variáveis: coeficiente de mortalidade infantil; coeficiente de mortalidade geral padronizada; percentual de analfabetos; percentual de domicílios permanentes chefiados por mulheres e média de pessoas residentes por domicílio.

Testaram-se as seguintes estratégias de modelagem nos modelos de uso e de predição da demanda: (i) variáveis de oferta sem ponderação pela densidade populacional; (ii) variáveis de oferta com ponderação pela densidade populacional; (iii) exclusão das áreas que agregam em cada estado os municípios com menos de $200 \mathrm{mil}$ habitantes, já que representam áreas agregadas que agrupam municípios distintos e não contíguos; e (iv) exclusão das áreas das regiões Norte, Nordeste e Centro-Oeste, uma vez que representam as áreas com pior qualidade dos dados de estatística vital. 


\section{Resultados}

As 134 áreas estudadas apresentavam grande heterogeneidade em relação às necessidades de saúde, à oferta de serviços e à qualidade dos dados. A mortalidade infantil apresentou uma expressiva variação: 12,7 óbitos por mil nascidos vivos no Município de Foz do Iguaçu, no Paraná, e 76,6 óbitos por mil nascidos vivos em Campina Grande, na Paraíba. O dado corrigido de mortalidade infantil guardou maior coerência com o grau de desenvolvimento sócio-econômico da região do que o coeficiente de mortalidade geral (Tabela 1). No coeficiente de mortalidade geral padronizado, observaram-se marcados problemas na qualidade dos dados em determinadas áreas. Por exemplo, o conjunto de municípios com menos de 200 mil habitantes do Estado do Amazonas apresentou o menor coeficiente (3,32 mortes por mil habitantes) a despeito de incluir municípios dentre os mais pobres do país. O maior coeficiente de mortalidade geral (18,75 por mil habitantes) ocorreu em São Luís, capital do Estado do Maranhão, pertencente à Região Norte. Esse coeficiente foi de 7,40 e 8,04 óbitos por mil habitantes nas cidades de São Paulo e do Rio de Janeiro, as mais desenvolvidas do país. As regiões Norte e Nordeste apresentam áreas com coeficientes de mortalidade inferior a 4 óbitos por mil habitantes.

Os indicadores de oferta também apresentam grande variabilidade nas regiões. O número de empregos (postos) médicos em ambulatórios por mil habitantes variou positivamente em relação ao grau de desenvolvimento sócio-econômico da região. A média de empregos médicos na Região Norte foi cerca da metade da média de empregos na Região Sul.

A média de leitos com vínculo com o SUS por mil habitantes $(1,93)$ é maior do que a média de leitos privados sem vínculo com o SUS $(0,44)$ no país e em todas as regiões. Entretanto, os indicadores de leitos com e sem vínculo com o SUS não guardam a mesma relação observada com os empregos médicos (Tabela 1). As regiões CentroOeste e Nordeste apresentam a maior concentração de leitos com vínculo com o SUS (2,36 e 2,34 por mil habitantes, respectivamente). O Nordeste apresenta a menor média de leitos privados sem vínculo com o SUS (0,31 por mil habitantes) e o Sudeste a maior (0,52 por mil habitantes).

A razão do valor pago por internação de curta permanência padronizada por idade e sexo também varia. Nas regiões Sul e Centro-Oeste os valores são maiores em relação à média nacional $(1,05)$, que corresponde ao valor médio da Região Sudeste, e menores do que a média nacional nas regiões Norte e Nordeste.
Inicialmente, compararam-se dois modelos de uso e predição da demanda: o primeiro sem a ponderação das variáveis de oferta pelo quadrado da densidade populacional da área e o segundo introduzindo-se essa ponderação. No primeiro modelo (Tabela 2, modelo 1 ), o teste de endogeneidade não foi estatisticamente significativo. Nesse teste, as variáveis de necessidades de saúde no modelo de uso apresentaram coeficientes com sinais negativos, fato que se repetiu no modelo de predição da demanda. A incorporação das variáveis de oferta ponderadas pela densidade populacional tornou o teste de endogeneidade estatisticamente significativo (Tabela 2, modelo 2), tal como previsto no modelo teórico de demanda. Neste segundo teste, tanto no modelo de uso como no de predição da demanda, o coeficiente geral de mortalidade padronizado por idade e sexo apresenta significância estatística e sinal positivo, indicando, como esperado, aumento das internações nas áreas de maior mortalidade geral. No entanto, a mortalidade infantil e a média de pessoas por domicílio permaneceram com sinal negativo, tal como no modelo 1.

O melhor desempenho do modelo com as variáveis de oferta ponderadas pela densidade populacional implicou a opção pelas variáveis ponderadas nas modelagens subseqüentes (Tabela 2, modelos 3 e 4 ).

Testaram-se, no modelo 3, as áreas das capitais dos Estados e os municípios com mais de 200 mil habitantes $(n=107)$. Nesse caso, o objetivo foi excluir da modelagem as áreas com grande heterogeneidade nas características da população, que foram construídas a partir da agregação dos municípios com menos de 200 mil habitantes em cada Estado, independentemente de sua localização geográfica. Nesse modelo, o teste de endogeneidade foi estatisticamente significativo e os preditores do uso foram o percentual de analfabetos e a média de pessoas por domicílio, ambos com sinal negativo.

Como a baixa qualidade dos dados de mortalidade, em particular nas regiões Norte e Nordeste, pode introduzir erro nas estimativas, repetiuse a modelagem com ponderação das variáveis de oferta apenas nas áreas das regiões Sudeste e Sul $(n=77)$. Nesse caso, o teste de endogeneidade não foi estatisticamente significativo. Além disso, o coeficiente obtido para a variável mortalidade geral, padronizada por idade e sexo, foi negativo, sugerindo que o resultado obtido para essa variável no modelo 2, que incorpora as 134 áreas geográficas, estava enviesado por erro de informação. 
Características da população de estudo.

\begin{tabular}{|c|c|c|c|c|c|c|}
\hline \multirow[t]{2}{*}{ Variável } & \multicolumn{5}{|c|}{ Região } & \multirow[t]{2}{*}{ Brasil } \\
\hline & Norte & Nordeste & Sudeste & Sul & Centro-Oeste & \\
\hline \multicolumn{7}{|c|}{ Necessidade de saúde } \\
\hline \multicolumn{7}{|c|}{ Mortalidade infantil } \\
\hline Mínimo & 23,50 & 18,27 & 15,41 & 12,70 & 17,21 & 12,70 \\
\hline Máximo & 66,70 & 76,60 & 55,60 & 23,60 & 29,54 & 76,60 \\
\hline Média & 38,90 & 44,31 & 24,4 & 17,40 & 22,48 & 29,50 \\
\hline \multicolumn{7}{|c|}{ Coeficiente geral de mortalidade padronizada } \\
\hline Mínimo & 3,32 & 3,88 & 6,2 & 5,97 & 6,91 & 3,32 \\
\hline Máximo & 9,82 & 18,75 & 9,77 & 8,94 & 9,80 & 18,80 \\
\hline Média & 7,39 & 9,36 & 7,78 & 7,25 & 7,59 & 8,00 \\
\hline \multicolumn{7}{|c|}{ Percentual de analfabetos } \\
\hline Mínimo & 13,00 & 14,00 & 9,00 & 9,00 & 13,00 & 9,00 \\
\hline Máximo & 34,00 & 39,00 & 19,00 & 17,00 & 20,00 & 39,00 \\
\hline Média & 22,10 & 25,06 & 14,00 & 12,90 & 16,60 & 17,50 \\
\hline \multicolumn{7}{|c|}{ Percentual de domicílios com chefe mulher } \\
\hline Mínimo & 10,80 & 17,23 & 15,61 & 14,60 & 12,55 & 10,80 \\
\hline Máximo & 30,20 & 31,85 & 30,14 & 32,40 & 27,11 & 32,40 \\
\hline Média & 19,90 & 23,70 & 21,37 & 20,70 & 20,72 & 21,60 \\
\hline \multicolumn{7}{|c|}{ Média de pessoas por domicilio } \\
\hline Mínimo & 4,00 & 4,00 & 3,00 & 3,00 & 4,00 & 3,00 \\
\hline Máximo & 5,00 & 5,00 & 4,00 & 4,00 & 4,00 & 5,00 \\
\hline Média & 4,44 & 4,23 & 3,89 & 3,57 & 4,00 & 3,99 \\
\hline \multicolumn{7}{|c|}{ Oferta de serviços de saúde } \\
\hline \multicolumn{7}{|c|}{ Leitos com vínculo com SUS por mil habitantes } \\
\hline Mínimo & 1,02 & 0,23 & 0,04 & 0,00 & 0,75 & 0,00 \\
\hline Máximo & 3,02 & 7,38 & 5,50 & 3,99 & 4,65 & 7,38 \\
\hline Média & 1,85 & 2,34 & 1,73 & 1,73 & 2,36 & 1,93 \\
\hline \multicolumn{7}{|c|}{ Leitos privados sem vinculo com SUS por mil habitantes } \\
\hline Mínimo & 0,00 & 0,00 & 0,00 & 0,00 & 0,02 & 0,00 \\
\hline Máximo & 1,43 & 0,97 & 1,85 & 1,56 & 1,33 & 1,85 \\
\hline Média & 0,42 & 0,31 & 0,52 & 0,42 & 0,49 & 0,44 \\
\hline \multicolumn{7}{|c|}{ Postos de trabalho médico em ambulatórios por mil habitantes } \\
\hline Mínimo & 0,12 & 0,25 & 0,32 & 0,39 & 0,24 & 0,12 \\
\hline Máximo & 1,93 & 2,55 & 2,45 & 2,83 & 1,55 & 2,83 \\
\hline Média & 0,57 & 0,83 & 1,26 & 1,14 & 0,79 & 1,02 \\
\hline \multicolumn{7}{|c|}{ Internação de curta permanência } \\
\hline \multicolumn{7}{|c|}{ Razão de internação padronizada por idade e sexo } \\
\hline Mínimo & 0,17 & 0,56 & 0,59 & 0,96 & 0,89 & 0,17 \\
\hline Máximo & 1,18 & 1,40 & 2,29 & 2,31 & 2,03 & 2,31 \\
\hline Média & 0,66 & 0,86 & 1,05 & 1,52 & 1,28 & 1,05 \\
\hline
\end{tabular}

\section{Discussão}

Este trabalho apontou a existência de limitações, no contexto brasileiro, do emprego da metodologia de Carr-Hill et al. 5 para alocação eqüitativa de recursos financeiros. O principal problema referiu-se aos indicadores de necessidades, preditores de internações hospitalares de curta permanência nesta metodologia, que sistematicamente apresentaram coeficientes com sinal negativo. Esses coeficientes, ao serem aplicados com a finalidade de alocação de recursos, resultarão em distribuição inversa às necessidades de saúde, pois nas áreas com piores condições de vida e saúde o modelo produzirá estimativas de valor dos recursos financeiros para financiar as 
Modelos de predição de demanda para internação de curta permanência no Sistema Único de Saúde (SUS), Brasil, 1999.

\begin{tabular}{|c|c|c|c|c|c|c|c|}
\hline \multirow[t]{2}{*}{ Modelos } & \multirow[t]{2}{*}{$\begin{array}{l}\text { Teste de } \\
\text { endogeneidade }\end{array}$} & \multicolumn{3}{|c|}{$\begin{array}{l}\text { Modelo final de uso } \\
\text { (ajustado pela oferta) }\end{array}$} & \multicolumn{3}{|c|}{$\begin{array}{l}\text { Modelo de predição da demanda } \\
\text { (sem ajuste pela oferta) }\end{array}$} \\
\hline & & Variável & B & Sig & Variável & B & Sig \\
\hline \multirow{7}{*}{$\begin{array}{l}\text { 1. Variáveis de oferta sem } \\
\text { correção por densidade } \\
\text { populacional (Brasil) ** }\end{array}$} & $F=0,85$ & Mortalidade infantil & $-9,67 \mathrm{E}-03$ & 0,003 & Mortalidade infantil & $-6,66 \mathrm{E}-03$ & 0,043 \\
\hline & $\mathrm{DF}=122$ & $\begin{array}{l}\text { Percentual de } \\
\text { analfabetos }\end{array}$ & $-1,63 \mathrm{E}-02$ & 0,031 & $\begin{array}{l}\text { Percentual de } \\
\text { analfabetos }\end{array}$ & $-1,15 E-02$ & 0,109 \\
\hline & $\mathrm{F}_{\mathrm{tab}}=2,68$ & $\begin{array}{l}\text { Média de pessoas } \\
\text { por domicílio }\end{array}$ & $-0,17$ & 0,021 & $\begin{array}{l}\text { Média de pessoas } \\
\text { por domicílio }\end{array}$ & $-0,193$ & 0,010 \\
\hline & & Leitos privados & $-0,22$ & 0,009 & & & \\
\hline & & Leitos SUS & 0,11 & 0,000 & & & \\
\hline & & $\begin{array}{l}\text { Postos médicos } \\
\text { em ambulatório }\end{array}$ & $-4,17 \mathrm{E}-02$ & 0,57 & & & \\
\hline & & $\mathrm{R} 2=0$ & ajustado $=$ & & $\mathrm{R} 2=0,27$ & justado $=0$ & \\
\hline \multirow{11}{*}{$\begin{array}{l}\text { 2. Variáveis de oferta } \\
\text { corrigidas por densidade } \\
\text { populacional (Brasil) }\end{array}$} & $F=4,98$ & Mortalidade infantil & $-9,56 \mathrm{E}-03$ & 0,006 & Mortalidade infantil & $-1,22 \mathrm{E}-02$ & 0,000 \\
\hline & $\mathrm{DF}=122$ & $\begin{array}{l}\text { Coeficiente de mortali- } \\
\text { dade geral padronizada }\end{array}$ & $3,25 \mathrm{E}-02$ & 0,104 & $\begin{array}{l}\text { Coeficiente de mortali- } \\
\text { dade geral padronizada }\end{array}$ & $4,18 \mathrm{E}-02$ & 0,017 \\
\hline & $F_{\text {tab }}=2,68$ & $\begin{array}{l}\text { Percentual de } \\
\text { analfabetos }\end{array}$ & $-1,27 \mathrm{E}-02$ & 0,082 & $\begin{array}{l}\text { Média pessoas } \\
\text { por domicílio }\end{array}$ & $-0,24$ & 0,001 \\
\hline & & Média pessoas & $-0,193$ & 0,007 & & & \\
\hline & & por domicílio & & & & & \\
\hline & & $\begin{array}{l}\text { Percentual de domicílios } \\
\text { com chefe mulher }\end{array}$ & $1,92 \mathrm{E}-02$ & 0,094 & & & \\
\hline & & Leitos privados $\left(P^{\star \star \star}\right)$ & $2,35 \mathrm{E}-08$ & 0,107 & & & \\
\hline & & Leitos SUS (P) & $-7,53 \mathrm{E}-09$ & 0,155 & & & \\
\hline & & Postos médicos & $-1,22 \mathrm{E}-08$ & 0,048 & & & \\
\hline & & em ambulatório (P) & & & & & \\
\hline & & $\mathrm{R} 2=0$, & ajustado $=$ & & $\mathrm{R} 2=0,29$ & justado $=0$ & \\
\hline \multirow{6}{*}{$\begin{array}{l}\text { 3. Variáveis de oferta } \\
\text { corrigidas por densidade } \\
\text { populacional (municípios) }\end{array}$} & $F=3,48$ & $\begin{array}{l}\text { Percentual de } \\
\text { analfabetos }\end{array}$ & $-2,62 \mathrm{E}-02$ & 0,004 & $\begin{array}{l}\text { Percentual de } \\
\text { analfabetos }\end{array}$ & $-1,86 \mathrm{E}-02$ & 0,043 \\
\hline & $D F=96$ & $\begin{array}{l}\text { Média de pessoas } \\
\text { por domicílio }\end{array}$ & $-0,20$ & 0,026 & $\begin{array}{l}\text { Média de pessoas } \\
\text { por domicílio }\end{array}$ & $-0,21$ & 0,023 \\
\hline & $F_{t a b}=2,68$ & Leitos privados $\left(P^{\star \star \star}\right)$ & $5,92 \mathrm{E}-09$ & 0,665 & & & \\
\hline & & Leitos SUS (P) & $-3,30 \mathrm{E}-09$ & 0,339 & & & \\
\hline & & $\begin{array}{l}\text { Postos médicos em } \\
\text { ambulatório }(P)\end{array}$ & $-6,39 \mathrm{E}-09$ & 0,281 & & & \\
\hline & & $\mathrm{R} 2=0$, & ajustado $=$ & & $\mathrm{R} 2=0,14$ & justado $=0$ & \\
\hline \multirow{8}{*}{$\begin{array}{l}\text { 4. Variáveis de oferta } \\
\text { corrigidas por } \\
\text { densidade populacional } \\
\text { (regiões Sudeste e Sul) }\end{array}$} & $F=2,37$ & Mortalidade infantil & $-1,33 \mathrm{E}-02$ & 0,042 & Mortalidade infantil & $-1,21 \mathrm{E}-02$ & 0,064 \\
\hline & $\mathrm{DF}=65$ & $\begin{array}{l}\text { Coeficiente de mortali- } \\
\text { dade geral padronizada }\end{array}$ & $-0,13$ & 0,036 & $\begin{array}{l}\text { Coeficiente de mortali- } \\
\text { dade geral padronizada }\end{array}$ & $-0,12$ & 0,030 \\
\hline & $F_{t a b}=2,68$ & $\begin{array}{l}\text { Percentual de domicílios } \\
\text { com chefe mulher }\end{array}$ & $2,60 \mathrm{E}-02$ & 0,073 & & & \\
\hline & & Leitos privados & $-8,18 \mathrm{E}-09$ & 0,312 & & & \\
\hline & & Leitos SUS & $-2,09 E-09$ & 0,417 & & & \\
\hline & & Postos médicos em & & & & & \\
\hline & & ambulatório & 1,79E-09 & 0,656 & & & \\
\hline & & $\mathrm{R} 2=0$, & ajustado $=($ & & $\mathrm{R} 2=0,14$ & justado $=0$ & \\
\hline
\end{tabular}

Nota: As variáveis de oferta foram leitos privados por mil habitantes, leitos públicos por mil habitantes e postos de trabalho médico em ambulatório por mil habitantes.

(P): valor predito

* Variáveis no modelo completo de uso: mortalidade infantil corrigida; coeficiente de mortalidade geral padronizada por idade e sexo corrigida; percentual de analfabetos; percentual de domicílios com chefe mulher; e número médio de pessoas por domicílio; 
internações menores do que nas áreas com melhores condições de vida.

Carr-Hill et al. 5 assumem que necessidade de saúde é um conceito complexo, pouco claro e incomensurável, mas que pode ser inferida através de características demográficas, sociais e de saúde. Dessa forma, inclui-se na modelagem do uso de serviços, além de variáveis demográficas e de saúde, um conjunto de variáveis sócio-econômicas, com a intenção de captar aspectos das necessidades não incorporados pelas variáveis de saúde. Esta decisão valida-se nas fortes evidências sobre a determinação social da saúde que indicam, em geral, um gradiente no qual as pessoas em pior condição social apresentam piores condições de saúde comparativamente às pessoas em melhor condição social 16 .

Apesar da consistência teórica, o modelo empírico de Carr-Hill et al. 5 apresenta uma característica que pode fragilizá-lo: empregar dados sobre a real utilização de serviços na estimativa do uso baseada nas necessidades de saúde da população. Dados sobre o uso de serviços de saúde são medidas imperfeitas da demanda aos serviços de saúde. O uso de serviços é função das necessidades, mas também da oferta de serviços e de outros fatores, particularmente fatores sociais, que podem gerar barreiras de acesso, na dependência das características gerais dessa oferta em cada lugar. Em situações particulares, como no Brasil, onde a demanda e o uso de serviços apresentam marcada variação social e as necessidades não atendidas são comparativamente maiores que as observadas no sistema de saúde inglês, as medidas de saúde e sociais tendem a expressar mais as barreiras de acesso do que as reais necessidades de saúde da população; isto é, a magnitude das desigualdades sociais no uso de serviços de saúde no Brasil faz com que, em modelos de predição do uso de serviços de saúde, as medidas de saúde e sociais reflitam menos as necessidades de saúde consideradas legítimas, expressando fundamentalmente as barreiras de acesso aos serviços de saúde.

No Brasil, como nos demais países, a condição social é importante determinante das condições de saúde, mas, contrariamente à Inglaterra, é também fator decisivo no uso de serviços de saúde. No Brasil, as pessoas socialmente menos favorecidas tendem a adoecer mais e usar menos serviços de saúde, o que caracteriza marcada iniqüidade no sistema de saúde brasileiro 9,10. Dados da PNAD/1998 mostram que a chance de uma pessoa avaliar negativamente o seu estado de saúde, ajustada por idade e sexo, aumenta na medida em que a sua escolaridade e renda diminuem. Inversamente, a chance de uso de serviços de saúde, ajustado por idade, sexo e ne- cessidade, diminui na medida em que a escolaridade e a renda decrescem ${ }^{1}$. Em resumo, no Brasil, os indivíduos com melhores condições sociais são mais saudáveis e usam mais serviços de saúde do que as pessoas com piores condições sociais.

A disponibilidade de leitos, médicos e outros recursos de saúde é fator necessário ao uso. Neste estudo, buscou-se verificar, dada a marcada desigualdade social no consumo de serviços de saúde no país, se o ajuste pela oferta de serviços na estimativa da demanda baseada em necessidades proposta pela metodologia de Carr-Hill et al. 5 minimizaria os efeitos dessa desigualdade nesta estimativa; isto é, a pergunta feita foi se a modelagem do uso padronizada pela oferta de serviços seria capaz de produzir estimativas de demanda coerentes com o padrão de necessidades da população. Analisaram-se as variáveis de oferta com e sem correção pela densidade populacional. A correção foi usada para buscar minimizar as distorções existentes na distribuição geográfica da oferta em relação à população. Essa correção tornou o teste de endogeneidade estatisticamente significativo, resultado este mais coerente com o modelo teórico. Testou-se também um modelo que empregou as variáveis de oferta corrigidas e que excluiu as áreas com menos de 200 mil habitantes, obtendo-se resultado semelhante; porém, a redução no número de áreas piorou o desempenho do modelo.

A incorporação das variáveis de oferta - não corrigida e corrigida - nas equações de uso de serviços de saúde não foi capaz de eliminar o efeito negativo das condições sociais e de saúde no uso de serviços de saúde no Brasil, o que corrobora a hipótese de que no país as desigualdades sociais no uso não são explicadas apenas por desigualdades na disponibilidade de serviços e que existem outras barreiras sociais no acesso aos serviços de saúde. Por exemplo, a capacidade de compra das famílias e a cobertura de planos de saúde teriam efeito independente sobre o uso de serviços de saúde no país. Outros estudos já mostraram que o padrão de internações no Brasil é fortemente influenciado pela cobertura de plano de saúde, com as pessoas com planos privados apresentando maior chance de internação 10,17 .

Problemas nos dados utilizados neste trabalho devem ser destacados, pois limitam a validade de alguns resultados. A instabilidade observada nos testes de endogeneidade e nos modelos de uso é um problema que pode estar referido ao pequeno número de áreas estudadas, à heterogeneidade sócio-demográfica e de saúde das áreas compostas pelo conjunto de municípios com população menor que 200 mil habitantes e 
à baixa qualidade de alguns dados, em particular, dos dados de mortalidade. A reduzida magnitude dos coeficientes de mortalidade geral padronizado por idade e sexo, observada particularmente em áreas das regiões Norte e Nordeste, é por si indicadora de precária qualidade dos dados 15. A baixa qualidade do dado de mortalidade geral obrigou o emprego de diferentes estratégias para avaliar seu impacto na modelagem. Os dados sociais mostram melhor qualidade, inferida a partir dos valores observados nas distintas regiões, que guardam coerência com os níveis de desenvolvimento sócio-econômico. Portanto, limitações dos dados disponíveis no momento da realização deste estudo também devem ser consideradas na interpretação dos resultados.

Os modelos de demanda testados não foram ponderados pela população, tal como na metodologia original. Nesta metodologia, a ponderação objetivou relativizar o padrão de uso das pequenas localidades na estimativa da média nacional de internações, usada para o cálculo da fórmula de alocação de recursos. A incorporação de áreas geográficas que agregam municípios com menos de 200 mil habitantes impediu essa ponderação, pois distorceu o tamanho relativo das populações entre áreas. Também não foi testado o efeito contextual das políticas de saúde locais e estaduais no padrão de uso de serviços que exigiria o emprego de modelagem hierárquica, como sugerem Carr-Hill et al. 5 .

A variável dependente utilizada neste estudo, ao basear-se em dados sobre gastos hospitalares, incorpora melhor a variabilidade dos tipos de pacientes internados do que a simples quantificação de internações. Sua construção requer informação que pode ser obtida dos valores usados pelo Ministério da Saúde para a remuneração das internações financiadas pelo SUS, contudo deixa de fora as hospitalizações financiadas pelos planos de saúde. Essa exclusão impacta a estimativa da demanda baseada em necessidades; entretanto, considera-se que a incorporação das internações financiadas pelos planos de saúde só tende a acentuar as desigualdades de acesso apontadas, já que a cobertura de planos de saúde no Brasil mostra-se positivamente associada ao uso de serviços de saúde 10 .

Uma outra limitação do estudo deve-se ao fato de que a política adotada para reajuste dos valores dos procedimentos hospitalares ao longo dos anos não guardou obrigatoriamente associa- ção com a complexidade do procedimento realizado. Assim, o valor do procedimento realizado não discrimina, com rigor, grupos de pacientes com consumo de recursos diferenciado.

A disponibilidade de dados em âmbito municipal, assim como de indicadores de saúde e sociais mais atualizados e mais específicos, possivelmente produziria estimativas mais consistentes. Estes testes não foram realizados, pois, no momento da elaboração do banco de dados empregado neste estudo, ainda não se contava com os dados do Censo Demográfico de 2000. Entretanto, o sinal negativo nos coeficientes de necessidades de saúde não parece estar associado aos problemas de qualidade dos dados. Os vários modelos testados foram consistentes nesse sentido, indicando ser esse um problema que, como apontado anteriormente, reflete principalmente a estrutura socialmente desigual do consumo de serviços de saúde no país.

Em síntese, os resultados deste estudo permitem concluir que o emprego de modelos de demanda baseados nas necessidades de saúde para distribuição de recursos financeiros entre áreas apresenta limitações em contextos com sistemas de saúde marcados por grandes iniqüidades no acesso, como o do Brasil. Portanto, novas alternativas devem ser exploradas para a estimativa de demanda baseada em necessidades em países com padrão de uso de serviços de saúde semelhantes ao do Brasil. Além disso, em face da limitação das informações disponíveis à época do desenvolvimento deste estudo, novas análises devem ser realizadas utilizando dados do Censo Demográfico de 2000, o que permitirá a incorporação de novas variáveis e o emprego de unidades de análise mais homogêneas, como o âmbito municipal. Assim, vale destacar a importância de se explorar a incorporação ao estudo de variáveis de morbidade percebida e referida (auto-avaliação do estado de saúde, prevalência de doenças crônicas e restrição de atividades rotineiras por motivo de doença, dentre outras) em modelos voltados para a alocação eqüitativa dos recursos financeiros do SUS.

Por fim, a metodologia inglesa aqui analisada, apesar de não aplicável para fins de alocação eqüitativa de recursos no país, pode ser um instrumento útil no acompanhamento do desempenho do SUS no que diz respeito ao acesso e uso de serviços de saúde em relação às necessidades de saúde da população. 


\section{Resumo}

No início da década de 1990, foi desenvolvida na Inglaterra uma nova metodologia para alocação de recursos financeiros aos serviços de saúde baseada nas necessidades de saúde da população. Essa metodologia caracteriza-se por adotar o modelo teórico de demanda aos serviços de saúde e por empregar dados de utilização dos serviços para estimar o uso com base na necessidade de saúde. Este artigo objetivou avaliar a aplicabilidade dessa metodologia para alocação de recursos federais no Brasil. Empregaram-se dados do Sistema de Informações Hospitalares do Sistema Único de Saúde sobre as internações de curta permanência ocorridas no Brasil em 1999. Foram analisadas 134 áreas geográficas, que cobrem o país como um todo. Os modelos estatísticos para estimar o uso testaram as seguintes variáveis de necessidades: mortalidade infantil; mortalidade geral padronizada; percentual de analfabetos; percentual de domicílios permanentes chefiados por mulheres e média de pessoas por domicílio. Todos os modelos de estimativa do uso de testados apresentaram coeficientes de regressão com sinal negativo, o que indica que a metodologia estudada apresenta restrições em contextos com grandes desigualdades sociais no uso de serviços de saúde, como o do Brasil.

Alocação de Recursos; Necessidades e Demandas de Serviços de Saúde; Eqüidade; Metodologia

\section{Referências}

1. Porto S, Vianna SM, Ugá MA, Vianna CM, Martins M, Lucchese PTR, et al. Metodologia de alocação eqüitativa de recursos. Relatório final de projeto Reforsus. Rio de Janeiro: Escola Nacional de Saúde Pública, Fundação Oswaldo Cruz; 2002.

2. Bloor K, Maynard A. Equity in primary care. York: Centre for Health Economics, University of York; 1995. (Discussion Paper, 141).

3. Barr A, Logan R. Policy alternatives for resource allocation. Lancet 1977; 1:994-7.

4. Goldacre MJ, Harris RI. Mortality, morbidity, resource allocation, and planning: a consideration of disease classification. BMJ 1980; 281:1515-9.

5. Carr-Hill RA, Sheldon TA, Smith P, Martin S, Peacock S, Hardman G. Allocating resources to health authorities: development of method for small area analysis of use of inpatient services. BMJ 1994; 309:1046-9.

6. Foster DP. Mortality, morbidity and resource allocation. Lancet 1977; 1:997-8.

\section{Colaboradores}

Todos os autores participaram de todas as etapas do trabalho.

\section{Agradecimentos}

Ao Dr. Carr-Hill por suas valiosas sugestões que ajudaram a aprimorar as análises realizadas; aos estatísticos Paulo Borges e Jurema Mota pela montagem dos dados; à Priscilla Mouta Marques pela revisão deste artigo, auxiliando-nos na elaboração de sua versão final. Ao Reforço à Reorganização do Sistema Único de Saúde (REFORSUS) do Ministério da Saúde pela subvenção à pesquisa.
7. Mays N. Geographical resource allocation in the English National Health Service. Int J Epidemiol 1995; 24:134-42.

8. Carr-Hill RA, Maynard AK, Slack R. Morbidity variation and RAWP. J Epidemiol Community Health 1990; 44:271-3.

9. Almeida C, Travassos C, Porto S, Labra E. Health reform in Brazil: a case study of inequity. Int J Health Serv 2000; 30:129-62.

10. Travassos CMR, Viacava F, Fernandes C, Almeida C. Desigualdades geográficas e sociais na utilização de serviços de saúde no Brasil. Ciênc Saúde Coletiva 2000; 5:133-49.

11. Viacava F, Bahia L. Oferta de serviços de saúde: uma análise da pesquisa Assistência Médico-Sanitária de 1999 (AMS 99). Brasília: Instituto de Pesquisa Econômica Aplicada; 2002. (Texto para Discussão, 915) 
12. Rice N, Smith P. Approaches to capitation and risk adjustment in health care: an international survey. York: Centre for Health Economics, University of York; 1999. (Occasional Paper, 38).

13. Departamento de Informática do SUS. Sistema de Informações Hospitalares do SUS. Tabela de procedimentos [CD-ROM]. Brasília: Ministério da Saúde; 1999.

14. Instituto Brasileiro de Geografia e Estatística. Estatística da saúde. Assistência médico-sanitária 1999. Rio de Janeiro: Instituto Brasileiro de Geografia e Estatística; 2000.
15. Szwarcwald CL, Leal MC, Andrade CLT, Souza PRB. Estimação da mortalidade infantil no Brasil: o que dizem as informações sobre óbitos e nascimentos do Ministério da Saúde? Cad Saúde Pública 2002; 18:1725-36.

16. Wilkinson R, Marmot M. Social determinants of health: the solid facts. Geneva: World Health Organization Regional Office for Europe; 2003.

17. Castro MSM, Travassos C, Carvalho MS. Fatores associados às internações hospitalares no Brasil. Ciênc Saúde Coletiva 2002; 7:795-811.

Recebido em 11/Dez/2005

Versão final reapresentada em 05/Dez/2006

Aprovado em 20/Dez/2006 